\title{
Initial findings of the impact of an 8-week intervention of sugar reformulated product exchange on cardiovascular risk factors
}

\author{
O. Markey ${ }^{1,2}$, J. Lejeune ${ }^{1,2}$ and J. A. Lovegrove $\mathrm{e}^{1,2}$ \\ ${ }^{1}$ Hugh Sinclair Unit of Human Nutrition, and ${ }^{2}$ Institute for Cardiovascular and Metabolic Research (ICMR), School of \\ Chemistry, Food and Pharmacy, University of Reading, Reading, RG6 6AP, UK
}

It has been reported from supply data that at a population level, every $628 \mathrm{~kJ} / \mathrm{d}(150 \mathrm{kcal} / \mathrm{d})$ increase in sugar availability, (equivalent to approximately one can of sugar-sweetened beverage daily), is independently associated with a $1.1 \%$ increase in diabetes mellitus prevalence $^{(1)}$. The WHO has recommended restricting non-milk extrinsic sugars (NMES) to $\leq 10 \%$ of $\mathrm{E}^{(2)}$. Reformulation of sugarcontaining processed foods is considered by some as a key option for achieving this target and for improving population health ${ }^{(3)}$. The impact of a sugar reformulated product exchange on cardiovascular risk factors was assessed using a randomised controlled, double-blind, crossover dietary intervention study.

Fifty healthy volunteers (16 male, 34 female; age 31.3 (SD 9.6) years; BMI 24.0 (SD 3.3) kg/m²) were randomly assigned to consume either unmodified or sugar reformulated foods and drinks, including juice, soft drinks, pasta sauces, baked beans, muesli, chocolate, ketchup and jam, for an 8-week period. This was followed by a 4-week washout period before volunteers crossed to the alternate intervention. The minimum target difference in dietary NMES intake between the unmodified and reformulated product exchange was $38 \mathrm{~g} / \mathrm{d}$. Fasting serum was collected pre-and post-intervention and used to determine glucose, lipid and inflammatory markers. Logarithmically transformed data were statistically analysed using ANCOVA.

There was no significant effect of the intervention on BMI. When adjusted for BMI, dietary restraint, age and gender there were no differences in fasting serum glucose, total cholesterol, TAG, NEFA or C-reactive protein (CRP) concentrations between interventions.

\begin{tabular}{|c|c|c|c|c|c|c|c|c|}
\hline & \multicolumn{2}{|c|}{ Diet A (Pre-) } & \multicolumn{2}{|c|}{ Diet A (Post-) } & \multicolumn{2}{|c|}{ Diet B (Pre-) } & \multicolumn{2}{|c|}{ Diet B (Post-) } \\
\hline & Mean & SD & Mean & SD & Mean & SD & Mean & $\mathrm{SD}$ \\
\hline BMI $\left(\mathrm{kg} / \mathrm{m}^{2}\right)$ & 24.0 & 3.4 & 24.1 & 3.3 & 24.1 & 3.3 & 24.1 & 3.4 \\
\hline Gluc $(\mathrm{mmol} / \mathrm{l})$ & 4.86 & 0.40 & 4.90 & 0.45 & 4.84 & 0.42 & 4.95 & 0.39 \\
\hline Chol (mmol/l) & 4.67 & 0.70 & 4.59 & 0.65 & 4.57 & 0.69 & 4.58 & 0.67 \\
\hline TAG $(\mathrm{mmol} / \mathrm{l})$ & 0.88 & 0.37 & 0.92 & 0.38 & 0.90 & 0.37 & 0.91 & 0.41 \\
\hline NEFA $(\mu \mathrm{mol} / \mathrm{l})$ & 465 & 164 & 443 & 190 & 474 & 176 & 437 & 170 \\
\hline CRP (mg/l) & 0.93 & 0.94 & 0.99 & 1.03 & 1.05 & 1.35 & 1.21 & 1.50 \\
\hline
\end{tabular}

Mean values with their standard deviations. For the purpose of on-going blinded analyses, the dietary intervention arms are coded as Diet A and Diet B (Pre- and Post-intervention).

Similar weight profiles after unmodified or sugar reformulated product consumption support the view that healthy volunteers may energy compensate whilst consuming sugar-reduced products which may have contributed to the lack of effect on cardiovascular risk factors. Further research is required to evaluate physiological and psychological compensatory mechanisms related to sugar reformulated product consumption.

This work was supported by Sugar Nutrition UK.

1. Basu S, Yoffe P, Hills N et al. (2013) PLoS ONE 8, e57873.

2. World Health Organization (2003) Diet, Nutrition and the Prevention of Chronic Diseases: Report of a Joint WHO/FAO expert consultation. WHO Technical Report Series, 916. Geneva: WHO.

3. van Raaij J, Hendriksen M \& Verhagen H (2009) Public Health Nutr. 12, 325-330. 